\section{Diffusion Pumps and Water Chillers}

\section{F.C. Thomas, Geological Survey of Canada (Atlantic) thomasf@agc.bio.ns.ca}

This note concerns two very important parts of most beam instrument systems; diffusion pumps and water chillers. As we'll see below, the two can be intimately connected.

Many SEMs, TEMs and other electron beam instruments contain one or more diffusion pumps as part of their vacuum systems. These are usually vertically-oriented cylindrical objects, perhaps $30 \mathrm{~cm}$ high, wrapped in several turns of copper tubing. They are usually placed behind or below the instrument's column, and typically handle high vacuums for tungsten filaments, or backing for ion pumps with other emitter types. Generally, these units are fairly maintenance-free; a change of oil every few years may be all that is required.

Because of the relatively high heat levels generated by diffusion pumps, most are liquid-cooled, hence the copper tubing. In most cases, the liquid is water, contained in and circulated by a separate water chiller unit. The chiller is simply a device containing a water reservoir, a cooling system, and a pump driven by an electric motor. Its cooling system is comprised of coils of copper tubing in the reservoir and a compressor

Water in the chiller is cooled to a thermostaticallycontrolled set point, and pumped via plastic or metal tubing directly to the beam instrument where it subsequently passes through the diffusion pump's copper tubes, absorbing heat in the process. In instruments with more than one diffusion pump, the water may simply be fed from the first diffusion pump di-

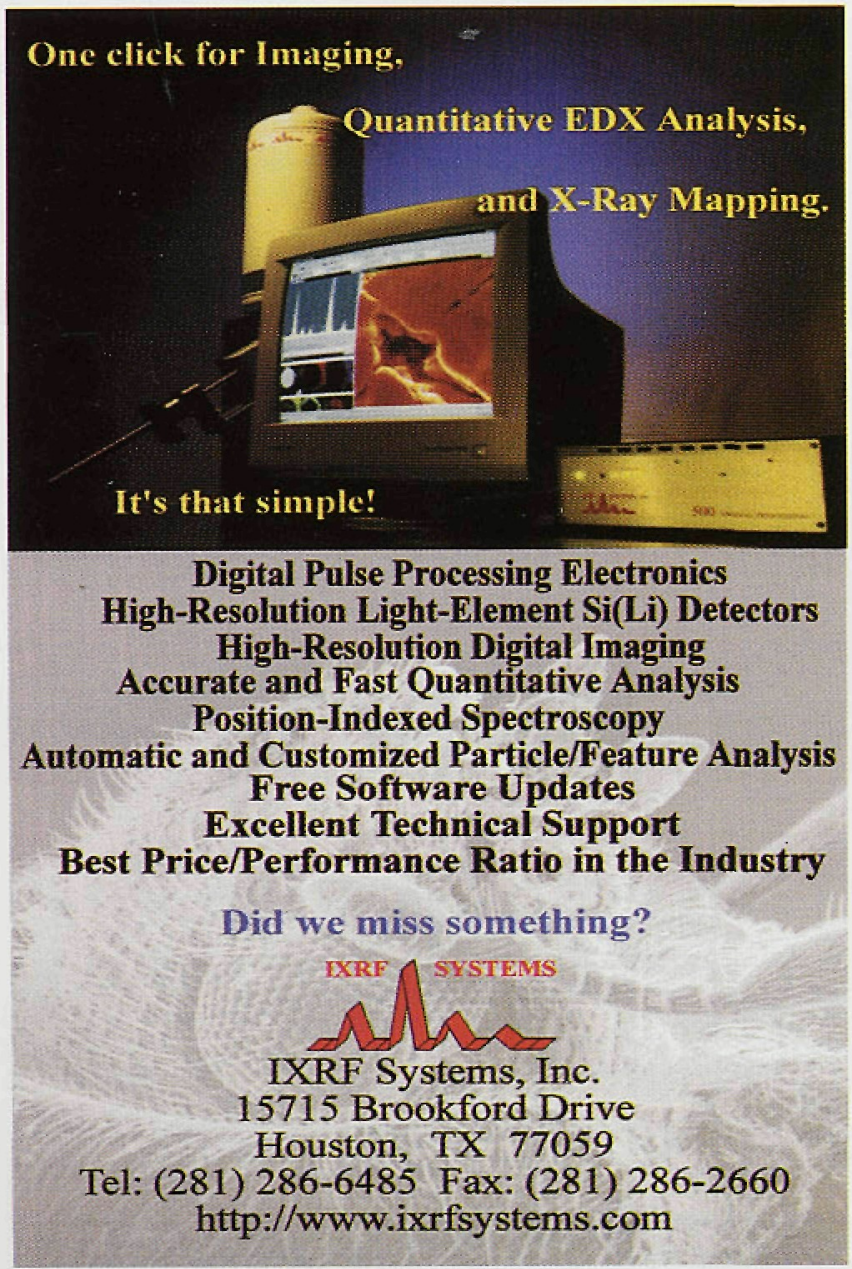

rectly to the other(s), before returning to the chiller.

Most diffusion pumps are equipped with some sort of heat sensor, designed to cut off power to the pump to prevent overheating. In most cases, the instrument will become inoperable at that point until the diffusion pump is back online. Should the diffusion pump trip off, the first thing to do is check the water flow. If the flow is reduced, one of two things has happened; either the chiller unit has failed, or the flow has become obstructed. If there is no flow-meter fitted to the line, the performance of the chiller must be checked the old-fashioned way. First make sure the beam instrument is in a safe mode, and would not be bothered by a sudden loss of water pressure. The gun may need to cool before proceeding. Then turn the chiller off and unplug it, open up the line from the chiller to the instrument at a convenient location, plug the unit back in and restart. The flow can be measured by timing how long it takes to fill a one-gallon vessel.

The manual for the beam instrument should say how much flow is required and at what temperature. If the flow from the chiller is good, repeat the whole procedure on the line from the beam instrument back to the chiller. This will determine if there is any blockage in the cooling lines in the instrument.

\section{Personal experience}

Recently I had a problem with diffusion pumps tripping off. When I checked the flow from the chiller to my SEM, it was good. I also had a look at the small built-in strainer in the chiller's water pump. It was fairly clean. However, when I checked the flow rate of water returning to the chiller (after having passed through the SEM), it was highly restricted. To flush the obstruction, I used a small burst of air from a pressurized can. The nozzle of the can fit quite nicely in the white plastic water line, and when a little air pressure was applied, a quantity of dirty water and small metallic bits came forcefully out of the end of the other line. I then repeated the process on the other line, to "back-flush".

Afterwards, when I reconnected all the water lines, flow was returned to normal and the diffusion pumps were able to stay properly cooled. Checking the $\mathrm{pH}$ of the water showed it be 5.3 , a bit acid. I had last filled the chiller about a year ago, while replacing a failed water pump. At that time I had simply used RO (Reverse Osmosis) water from our institute's source. Checking our fresh $\mathrm{RO}$ water right from the spigot showed the $\mathrm{pH}$ to be about 5.8 - better, but still on the acid side. No doubt the acidity of the water had contributed to the corrosion I was seeing

\section{Corrosion}

I was curious about the nature of the metallic bits l'd flushed from the system. Most of them looked bluish-green. Also, while I had the lines apart, I'd changed the in-line filter we use. It is one of those white, cylindrical, domestic-type filters, some $6.5 \times 23.5 \mathrm{~cm}$. It was noticeably green, also. Since the SEM was back on line, I was able to run EDS analyses on the metallic grains and on the green outside layer of the filter. Both showed large copper peaks, presumably from copper oxides. This was disturbing news, since it indicated that either the cooling coils in the chiller, or the copper tubes in the SEM, or both, were corroding. Since corrosion will normally occur quicker in warmer sites, it was more likely associated with the diffusion pumps.

In systems where both copper and iron fittings are present, the iron will normally corrode first. Stainless steel, of course, is somewhat more resistant, but can still corrode in some settings. Some colleagues have found that corrosion effects in water lines can be worst where tube size reduction fittings or right-angled elbows are present in the line. This can be attributed to increased erosion because of irregularities in flow patterns. Also, such spots 
are a prime location for loose granules of corrosion products to concentrate and further restrict flow. That is why "back-flushing" or temporarily forcing water through from the opposite direction can quickly clear a blockage.

\section{Chiller maintenance}

Recirculating water chillers often just don't get enough attention: they do their work quietly and unobtrusively, and it is easy to forget that they are there. This is especially true when, as in many situations, the chiller is not in the same room as the beam instrument, but is tucked away in a separate enclosure with the rotary pumps. If the chiller doesn't spring a leak or start making a noise, one may not notice anything is amiss until the diffusion pump starts giving trouble.

The most common problems associated with chillers are probably motor failure, pump failure and water quality. The first two are usually easy to pin down; unusual noises often start when the motor or pump is failing, and water leaks may develop at the motor/pump interface. Perhaps the most common type of failure in electric motors is excessive wear on the bearings. Check the manual to see if the motor has sealed bearings (ones that you should "never" need to lubricate) or if it requires periodic lubrication. If it does need lubrication, it is usually a matter of a drop or two of machine oil to be fed into indicated spots, usually on an annual or semi-annual basis. In most cases, failed motors and pumps can be easily repaired or replaced.

Deteriorating water quality is more insidious. It may happen over a period of weeks or even months. Biological growth (bacterial, algal, etc.) is a common problem in many systems; but tends to be less so where non-translucent lines are used. Acidification may result from such growth, or even from exposure to the air, as in the top of the chiller's reservoir.

Various chemicals can be added to the water in the chiller to address these problems. In chillers with an open reservoir (one in which a lid can be removed, revealing the whole tank and its cooling coils) many users sprinkle a bit of dichlorophene on the surface to inhibit biological growth. Others use glycol for the same purpose. Acid water can be buffered up to a neutral $\mathrm{pH}$ with sodium bicarbonate. Other commercially-sold preparations such as Aqua-Treet 42 may be used to inhibit corrosion.

Such additives should be used very judiciously, however. Glycol, for instance, can adversely affect certain types of seals in some instruments. Also, as it decomposes it may provide free carbon for uptake by biological agents, and so promote certain types of growth. Some chiller manufacturers may specify (and supply) small packets of their preferred anti-biological treatments. Before adding anything to the cooling water, check with the manufacturers of both the chiller and the beam instrument to make sure such additives are compatible with both units.

A small amount of preventative maintenance for the chiller will go a long way towards keeping the system trouble-free. Develop a monthly maintenance protocol which involves checking the level, appearance, and $\mathrm{pH}$ of the water in the system. For $\mathrm{pH}$, generally the closer to neutral the better. If there is an in-line filter present, check its condition. Also, check the flow rates, with flow meters or visual observation. Look for water leaks, and inspect the appearance of all water lines and hoses. Avoid unnecessary kinks or bends in the lines. Once or twice a year it may also be a good idea to check the strainer in the chiller's pump, if one is present. Take the time to listen for a moment to learn what a normal, healthy chiller should sound like. Changes will then quickly reveal themselves.

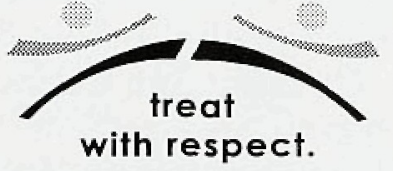

\section{Working in the presence of Nobel Laureates ifn't fomething you get to do every day? Why not?}

You give so much to others. When was the last time you checked your own direction? At UCSF Medical Center, we boast one of the most talented, educated and dedicated staff in healthcare as well as an amazing benefits package including comprehensive medical, dental and vision plans, life insurance, 4036 retirement plan, 15 paid vacation days in the first year, 13 paid holidays, and 12 sick days. Consider joining our team of professionals in this role:

\section{ELECTRON MICROSCOPY TECHNOLOGIST}

You will serve as a technical expert performing diagnostic electron microscopy on a wide variety of specimens, immunofluorescence microscopy, muscle histochemistry staining and nerve tease preparations. Will also assistadvise faculty in planning and completion of research projects. Requires a college degree in a biological science, chemistry or a related field or equivalent education and work experience. Must have 3 years in a hospital pathology laboratory performing all aspects of diagnostic electron microscopy work-up, extensive knowledge of tissue ultrastructure, including Uitrastructural pathology, knowledge of electron microscopy, and excellent interpersonal and communication skills. Experience in muscle histochemistry highly desirable. EMSA Certification in Electron Microscopy with formal training in electron microscopy is strongly preferred.

Interesied candidates can send resume to: jobs@ucsfmedctrorg or fax (415) 3534690. EOE.

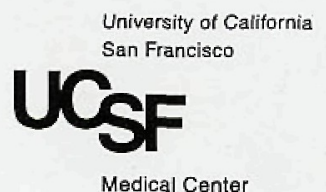

www.ucsfhealth.org/jobs

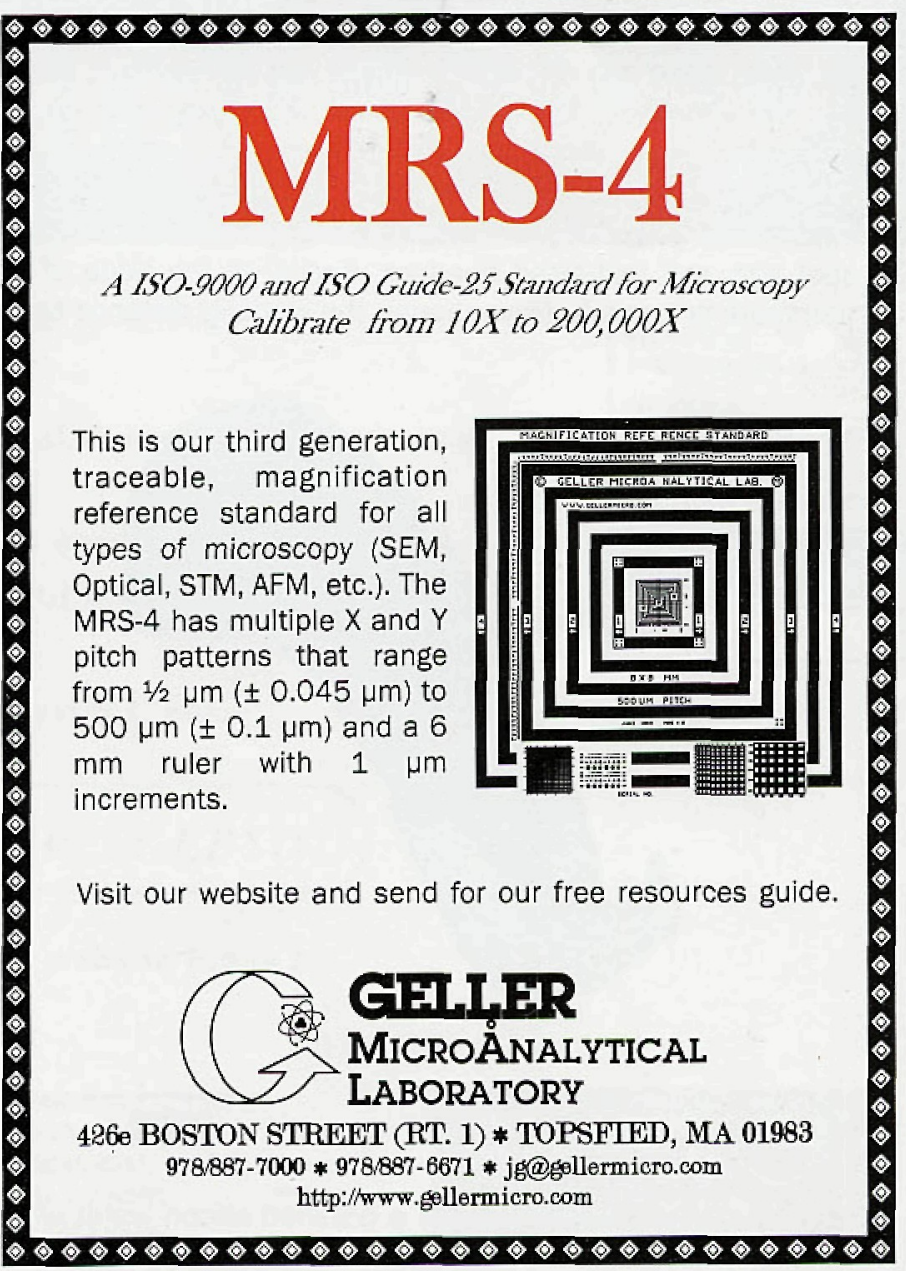

